\title{
A new modulation approach to decrease total harmonic distortion of the SPWM voltage waveform using genetic optimization technique
}

\author{
N. Tutkun ${ }^{1}$ \\ ${ }^{1}$ Department of Electrical \& Electronic Engineering \\ Faculty of Engineering, Zonguldak Karaelmas University \\ Incivez, 67100 Zonguldak (Turkey) \\ Phone/Fax number:+0090 372 2574023, e-mail: tutkun@karaelmas.edu.tr
}

\begin{abstract}
The pulse width modulated power inverters have been increasingly using to convert $\mathrm{DC}$ power to $\mathrm{AC}$ power in small wind plants. When these inverters are used for power conversion, the integrated output voltage waveform is inevitably distorted. Although increasing the switching frequency is one choice to achieve the smoother output voltage, it adds extra harmonics to the output. There are various techniques such as analogue filtering, harmonic elimination etc. to reduce the number of harmonics usually affecting the system performance. In this study, a simple but efficient modulation approach based on the optimization of the shape of the triangular voltage waveform is proposed. The results have shown that the total harmonic distortion of the optimized voltage waveform decreases gradually and thus helps improve on the power quality during the conversion.
\end{abstract}

\section{Key words}

Total harmonic distortion, pulse width modulation, harmonic losses, power electronic devices, genetic algorithms.

\section{Introduction}

The pulse width modulated (PWM) inverters are widely used for converting DC power to AC power in low power wind plants [1]. When such inverters are employed for power conversion, the integrated output voltage almost close to sinusoidal waveform but contains several harmonics affecting the power quality inevitably [2],[3]. The one solution to this problem is to increase the switching frequency for obtaining smoother output waveform being very close to sinusoidal one. However, increasing the switching frequency enlarges the number of harmonics as well as leads to additional switching losses. The number of harmonics can be reduced to that being less than normal provided that previously and newly developed techniques are implemented.

In this study, a new modulation technique to reduce the number of harmonics through the genetic optimization technique (GOT) is proposed. The modulation technique is based on optimization of the triangular waveform to generate PWM waveform containing less harmonic distortion (THD) for certain modulation index, switching frequency and operating frequency. By optimizing triangular waveform, the THD of the PWM waveform is less than that of the regular PWM one. Although this approach has not been tested at large, the THD has been achieved to decrease by almost $10 \%$.

\section{Overview of the SPWM Output Voltage}

The sinusoidal PWM (SPWM) voltage waveform is obtained by comparing a carrier (usually triangular) and a reference (usually sinusoidal) voltage waveform. When the resultant waveform is integrated over a period, the output voltage waveform which is similar to sinusoidal voltage waveform is acquired as shown in Fig. 1.

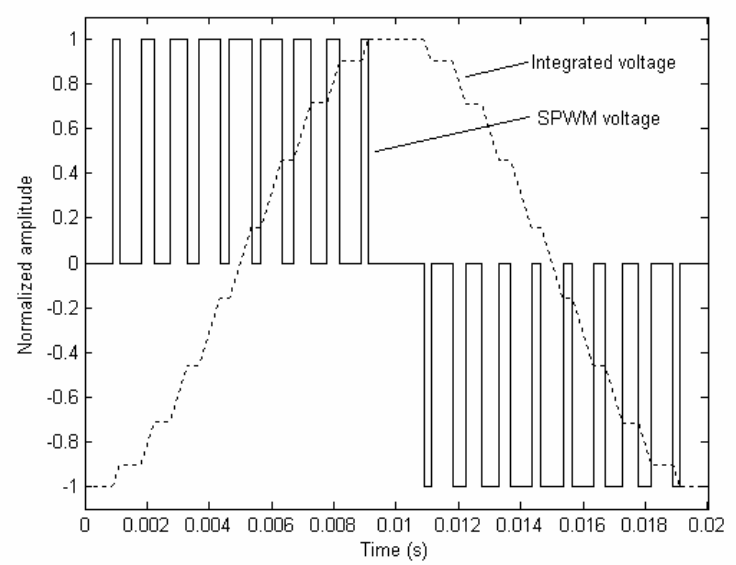

Fig. 1. A typical SPWM and integrated voltages with 9 pulses per half cycle.

\section{A. Definition of the Asymmetrical Triangular Approach for the SPWM}

The THD can be decreased by optimizing the triangular waveform on condition that the switching frequency of the triangular waveform is unchanged. In conventional modulation techniques, the triangular waveform is 
usually symmetrical and harmonics of the SPWM output voltage are mostly dependent of frequency of triangular voltage waveform. In this proposed approach, the triangular waveform is globally optimized to make the THD less than symmetrical one. A typical triangular waveform is composed of two parts: one is a line with a positive slope; the other is a line with a negative slope as depicted in Fig. 2. They are defined in intervals of 0 to $T_{1}$ and $T_{1}$ to $T_{2}$ respectively. In the optimization of the triangular waveform, coefficient $\alpha$ is defined as a single variable and varies from 0.1 to 1 . For instance, if the coefficient is 0.5 then a symmetrical triangular voltage waveform is generated. The definition of a typical triangular waveform for a single positive pulse can be given by

$V_{c}(t)=\left\{\begin{array}{cc}\frac{V_{c m}}{T_{1}} t & 0 \leq t \leq T_{1} \\ -\frac{V_{c m}}{T_{2}} t+V_{c m}\left(\frac{T_{1}}{T_{2}}+1\right) & T_{1} \leq t \leq T_{2}\end{array}\right.$

where $V_{c m}$, and $T_{1}$ and $T_{2}$ are the peak value of the triangular voltage, and time intervals for line function respectively.

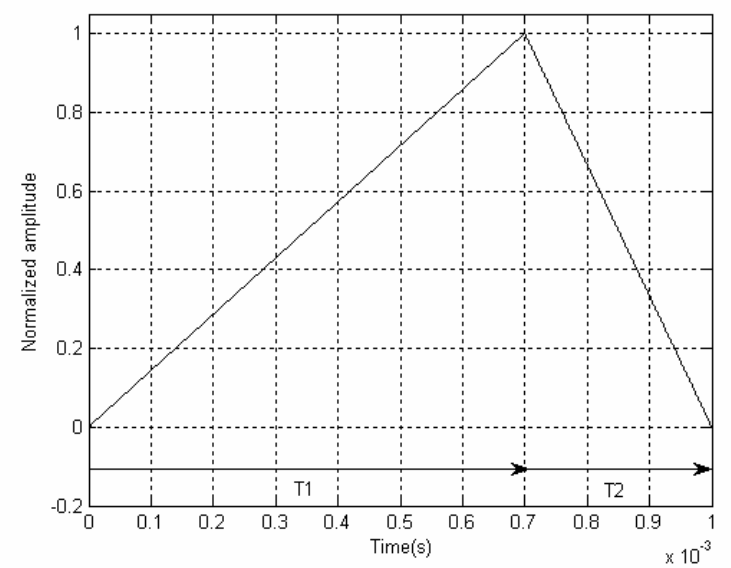

Fig. 2. An optimized single triangular waveform to obtain less THD.

\section{B. Optimization of SPWM Waveform with the Genetic Algorithms}

The proposed approach aims to optimize the triangular waveform through the search for the best using the real coded genetic algorithms (RCGA) method. In the RCGA software, coefficient $\alpha$ is defined as the input variable and the THD is the output variable. Each THD value is subtracted from constant A which is equal to maximum fitness to construct the fitness function $\mathrm{F}$ given by

$$
F=A-\frac{1}{V_{1}}\left(\sum_{n=2}^{\infty} V_{n}{ }^{2}\right)^{1 / 2}
$$

where $V_{1}$ and $V_{n}$ are the fundamental and $n^{\text {th }}$ harmonics of SPWM output voltage waveform respectively.
Optimization process was carried out by randomly creating a population with 100 individuals. Each individual was linearly mapped to a real number between 0.1 and 1 . Fitness value of each individual in the current population was calculated by (2) for selection based on the roulette wheel mechanism [4]. According to fitness values most fit individuals had a chance to be copied into the mating pool for implementing crossover and mutation operations.

Once the selection was completed, a single point crossover operation was put into effect on the selected individuals with probability of 0.65 and the jump mutation was applied to the current population with the probability of 0.05 . Finally, a new generation was created after all and the fittest individual was stored following each generation, in other words, the elitism was applied. The genetic process was repeatedly carried out until the 100 generations were encountered. The major steps of the algorithm are summarized below and the flowchart of a simple genetic process is shown in Fig. 3.

Step 1: Randomly create a population with individuals Step 2: Evaluate fitness of each individual

Step 3: Select parents according to their fitnesses

Step 4: Crossover selected population with crossover probability of 0.65

Step 5: Mutate a new population with mutation probability of 0.05

Step 6: Stop iteration if satisfied, else go to Step 2 and continue to carry out process until 100 generations are met.

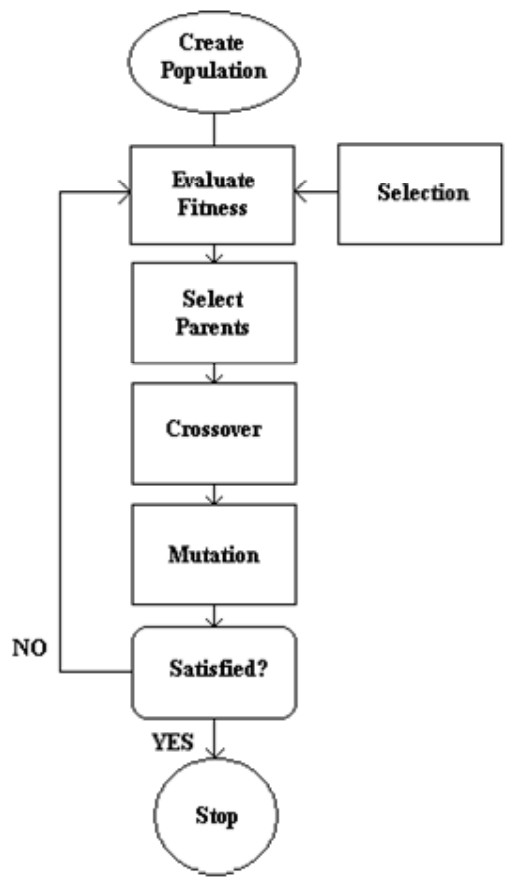

Fig. 3. A simple genetic algorithm process.

\section{Experimental Method}

The proposed algorithm was applied to make a single phase full-bridge voltage source PWM inverter circuit as shown in Fig 4. The four IGBT elements were used to 
switch DC power input to acquire pulses with constant amplitude and variable widths. In order to make the IGBTs on state within required time the PIC microprocessor and its ICs drivers were employed. To avoid increasing switching losses few capacitors were connected in the output of the ICs, provided that they change steep sided pulses to round sided ones. Besides, a very small duration was added to the output signal to avoid overlapping it following each cycle.

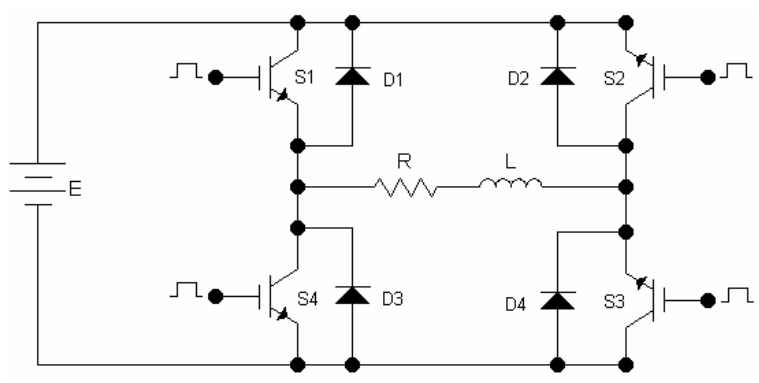

Fig. 4. A single phase SPWM inverter circuit under RL load.

\section{Results and Discussion}

Several simulation experiments were carried out to obtain best optimized triangular waveform for a certain modulation, the switching frequency and the operation frequency. Coefficient $\alpha$, the modulation index, the carrier frequency and the operating frequency varied from 0.1 to $1,0.5$ to 0.9 and 0.5 to $10 \mathrm{kHz}$ and 25 to $100 \mathrm{~Hz}$ respectively during the experiments. For each case, the THD value was calculated and compared with the ones obtained from the symmetrical triangular waveform. Fig 4 shows a comparison of the THD obtained from $1 \mathrm{kHz}$ optimized triangular waveform with the symmetrical one and the horizontal axis compares the modulation indices with the THDs in both waveforms.

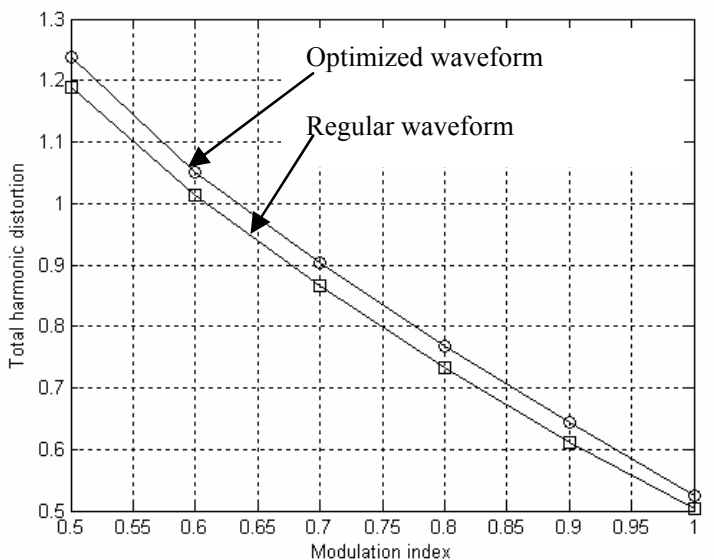

Fig. 5. Comparison of the THDs at the carrier frequency of 5 and $10 \mathrm{kHz}$.

According to Fig 5, the THD with the optimized triangular waveform is less than that with the symmetrical one and the actual average percentage difference between two waveforms is around 4\%. As the modulation index increases, the THD shows almost linear variation. The largest THD difference between both waveforms is $5 \%$, in other words, minimum THD is 0.6104 for the optimized coefficient $\alpha$ of 0.8592 and the modulation of index of 0.9 . With these optimized values, the frequency spectrum of the SPWM output voltage is illustrated in Figs 6 and 7 for typical comparison. As seen in Fig 7, the fundamental component of SPWM output voltage is slightly larger than 0.9 . This may simply help reduce the THD and improve power quality. According to Fig 7, the largest harmonic was 0.2 at $2 \mathrm{kHz}$ and other harmonics remained almost the same with other frequency spectrum shown in Fig 6.

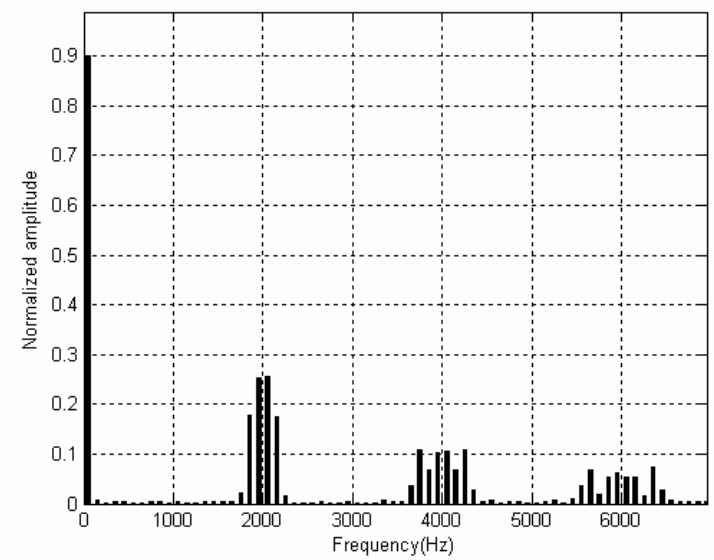

Fig. 6. Frequency spectrum of regular SPWM voltage at modulation index of 0.9 .

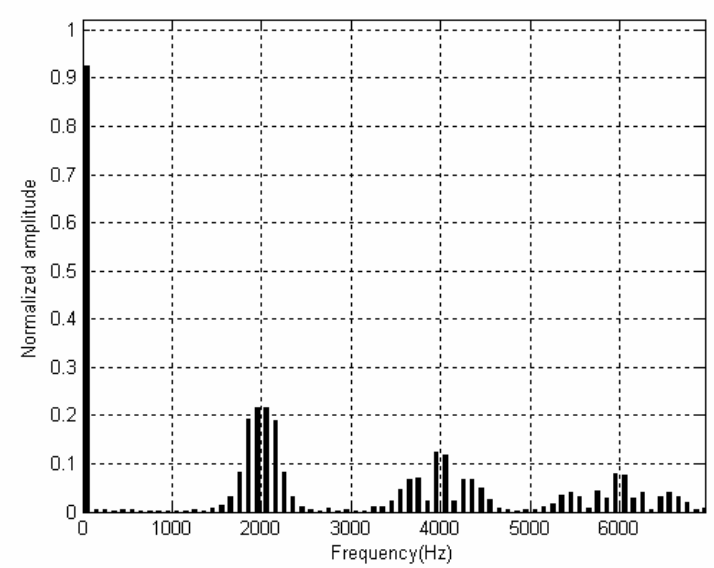

Fig. 7. Frequency spectrum of optimized SPWM voltage at modulation index of 0.9 .

In order to see effects of the carrier frequency on the THD and its frequency spectrum, the genetic program was run for those frequencies of $2,3,4,5,10 \mathrm{kHz}$ and it produced similar outputs at $2,3,4 \mathrm{kHz}$ with those at 1 $\mathrm{kHz}$. The optimized THD at the switching frequencies of 5 and $10 \mathrm{kHz}$ surprisingly increased at the modulation indices being larger than 0.7 . It should be noted that at these switching frequencies, the THD difference between the symmetric and optimized triangular waveforms increased by almost up to $29 \%$ around the modulation index of 0.7 and this is quite meaningful and encouraging outcomes. 
As it can be seen from above figure the carrier frequency and the modulation index of the SPWM voltage waveform may be very influential on power quality during the power conversion. In fact, the THD showed very small effects on power quality although the modulation index showed a great effect on it. This may seem to be important to SPWM inverter designers to obtain better power quality in wind plants during the conversion.

\section{Conclusion}

The THD in the SPWM voltage waveform can be decreased by simply optimizing the triangular voltage through the GOT hence; power quality may be improved in a sufficient level. The modulation index is the most influential parameter to decrease the number of harmonics in the integrated output voltage waveform. Minimum THD repeatedly occurs at the modulation index of 0.9 at all the carrier frequencies.

\section{References}

[1] K. L. Shi and H. Li, "Optimized random PWM strategy based on Genetic Algorithms", IEEE Trans. Mag. Vol. 33, pp. 07-11, 2003.

[2] M. H. Rasid, Power Electronics:circuits, devices, and applications, Prentice Hall, New Jersey (1993), pp. 359410.

[3] M. T. Briickner and D. G. Holmes, "Optimal Pulse Width Modulation for Three-Level Inverters", IEEE Trans., Vol. 33, pp. 165-170, 2003.

[4]..D. E. Goldberg, Genetic algorithms in search, optimization \& machine learning, Addison-Wesley, Reading, (1989), pp. 59-88. 